\title{
Nondestructive Evaluation of Solids by Photothermal Interferometry on Nonreflective Surfaces
}

\author{
Z. Sodnik and H.J. Tiziani \\ Institut für Technische Optik, Universität Stuttgart, \\ Pfaffenwaldring 9, D-7000 Stuttgart 80, Fed.Rep. of Germany
}

\section{INTRODUCTION}

For nondestructive material evaluation (NDE) ultrasonic and $X$-ray methods have been well established for many years. About two decades ago an old effect, discovered by BELL $/ 1 /$, was theoretically examined by WHITE $/ 2 /$. A comprehensive theory for NDE utilizing a laser heat source was given by ROSENCWAIG et al./3/. It is called the thermoacoustic effect and describes the phenomenon of sound generation, when periodically heating the surface of a body with an audio frequency. Since heat generation is usually done optically, using a modulated laser beam or a laser pulse $14 /$, the process is called photoacoustic or photothermal depending on whether the main interest is in the acoustic or the thermal aspect respectively. One should however keep in mind that all effects happen at the same time. At first, light is converted into heat, which heats the surrounding air and generates sound. Second, heat causes thermal expansion, leading to sound transport in the material. We decided to call our experiment a photothermal process, although both effects are involved.

In a photothermal process the absorption of modulated light modifies the surface temperature of a specimen and causes a heat diffusion, called a thermal wave, to propagate through the material. Inhomoceneities in the material can be detected by directly /5/ or indirectly monitoring the thermal wave. In our indirect measurements we detect the periodically changing thermal expansion - caused by a modulated laser source - interferometrically.

Changes in the amplitude or the phase of the expansion, with respect to the temperature modulation, lead to the detection of subsurface defects.

\section{EXPERIMENTAL ARRANGEMENT}

A chopperwheel (CP) modulates the intensity of an argon laserbeam (L), which is focussed onto a specimen's surface, generating a temperature variation and a periodical spherical heat flow into the material as shown in Fig.1.

The specimen (SP) is mounted onto a stepper-motor-driven and computercontrolled table $(T)$, which can be moved in $X$ and $Y$ directions with a position accuracy of one micrometer.

To avold polishing of a surface we developed a tactile interferometrical sensor head (SH). After beam expansion (BE), spatial flltering (SF), and passing a beam splitter (BS), one beam of the interferometer, normaliy focussed onto the specimen 's surface, is now focussed onto a small steel pin, which is in mechanical contact with the specimen's surface. In order not to damage the surface or to misalign the interferometer, the sensor is removed 


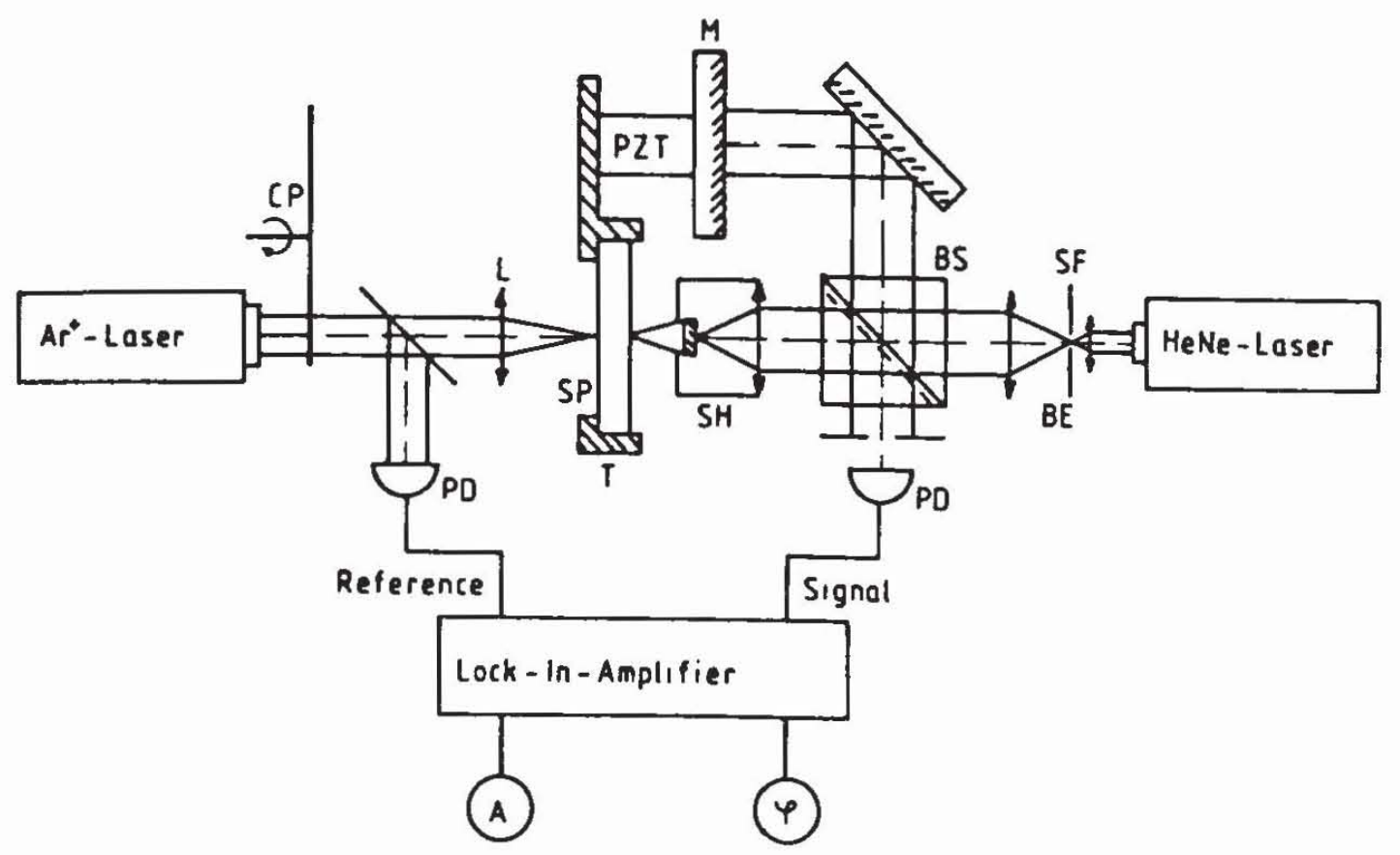

Figure 1: Experimental arrangement using a tactile sensor head

from the surface by an electromagnet, while moving the specimen to the next scanning position. The pin is attached to the focussing lens, its function is to transmit the specimen's surface expansion to a smail mirror, which is glued to its back.

For stability reasons it was found useful to have the reference bearn reflected back from a mirror (M), which is attached close to the specimen. In this way vibrations have less effect on the optical path difference in the interferometer. A computer-controlled piezo element (PZT) moves the reference mirror in order to adjust the interferometer.

The detection takes place on the rear surface of the specimen, opposite to the argon laser focus. It is also possible to generate and detect thermal waves on the same side of the specimen $/ 6,7 /$, using the effect of thermal waves being "reflected" at subsurface defects.

The zero order interference fringe is observed by a photodiode (PD) and its signal is fed into a lock-in amplifier. In amplitude and phase measurements subsurface defects can be detected.

\section{THEORETICAL CONSIDERATIONS}

Heat conduction in solids is a three-dimensional diffusion phenomenon $/ 8 /$, which can be solved for a thermal point source in an infinite homogeneous body. Due to the fact that expansions are measured, rather than radiation, our theory had to rely on a quasi-static approximation in the periodic case, under consideration of boundary conditions. We ignored the size of the argon laser focus spot because it is much smaller than the thermal wavelength and found a one-dimensional model in good agreement with the results. Assuming $T$ to be the amplitude of the temperature variation at the argon laser point of impact, $C$ the thermal expansion coefficient, $\mu$ the thermal diffusion length and ignoring the time dependence, then the expansion $\delta$ in a distance $l$ from the heat source was found to be 


$$
\delta(1)=\frac{C T}{1^{2}} \int_{0}^{1} x^{2} \operatorname{ExP}\left[-\frac{x}{\mu}(1+i)\right] d x
$$

\section{EXPERIMENTAL VERIFICATION OF THEORETICAL RESULTS}

To verify the theory, an aluminum wedge of a thickness variation between 150 to 2550 micrometers was examined using two different modulation frequencies. The theory (dashed line) predicts an unexpected result, that the amplitude decreases at the thin end of the specimen, but it cannot properly explain the results at its very thin end (at $20 \mathrm{~Hz}$ ). Equation (1) is basically developed from the theory of an infinite body, and a major difference occurs at the thin end of the wedge. With increasing thickness of the specimen or at a nigher frequency, both phase and amplitude follow the theoretically predicted variations.
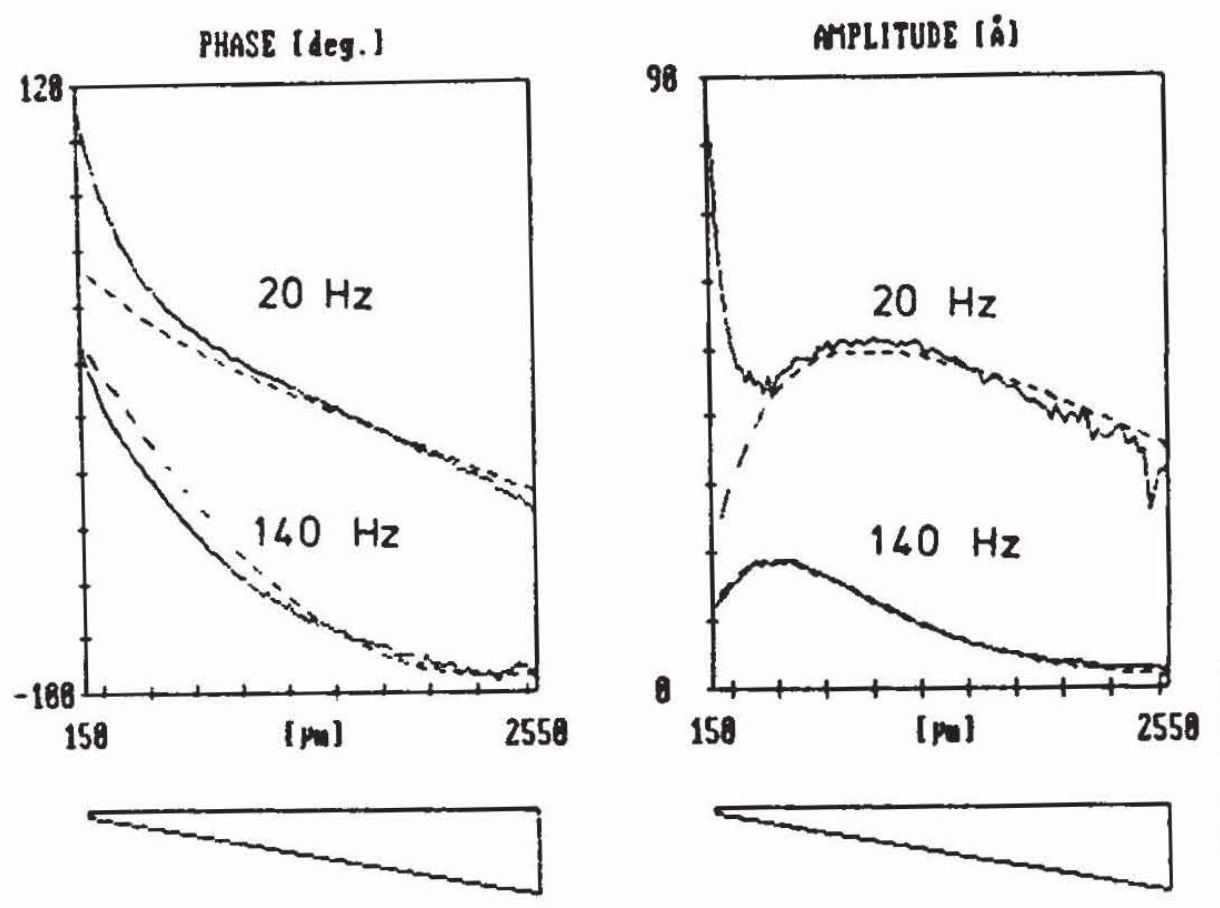

Figure 2:

Experimental and theoretical (dashed) results obtained on a wedge

An example for subsurface defect detection is shown in Fig.3. The specimen is a block of aluminum of $2.5 \mathrm{~mm}$ thickness, with three drilled holes of $0.8 \mathrm{~mm}$ diameter. The gap between the first and the second hole is $0.8 \mathrm{~mm}$ wide and between the second and the third $0.4 \mathrm{~mm}$ wide. The upper graphs are results obtained at a modulation frequency of 20 and the lower of $140 \mathrm{~Hz}$. Additionally a comparison is given between results obtained with an interferometer focussed onto the specimen's surface (upper curve) and one using the tactile sensor head (lower curve). As one can see the sensor head causes a phase decay of about 5 degrees, an amplitude damping of about $10 \%$ and a small increase in amplitude's noise. The phase response however, which is most important for the detection of subsurface defects, because unlike the amplitude it is not dependent on varying argon laser absorptions, is in both cases the same. This indicates, that there is no obvious loss in resolution. The width of the photoacoustic signal, being larger than the width of the holes, has its origin in the detection of expansions, which are influenced by the presence of a defect in a bigger radius then given by the thermal diffusion length. 
PHASE [deg.]
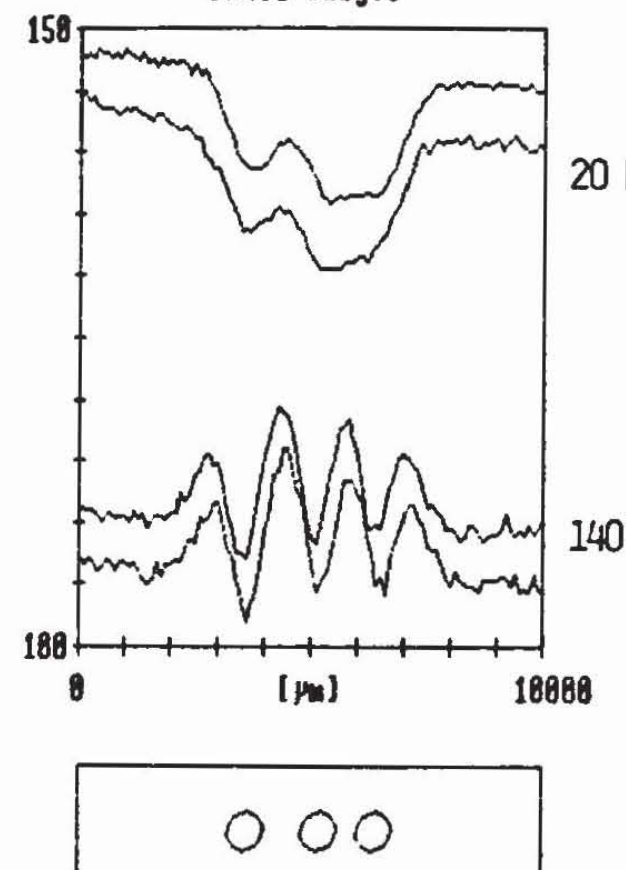

MPLITUDE [ด̊]
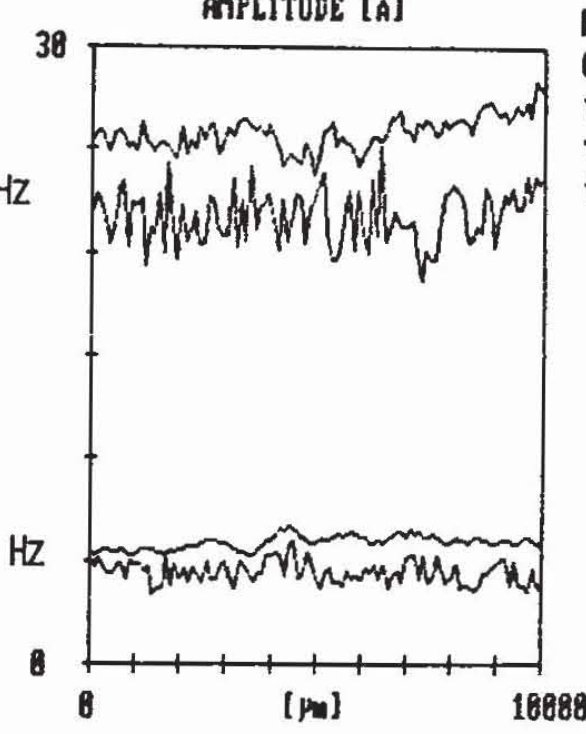

000
Figure 3:

Comparison between tactile and non-tactile subsurface defect detection

\section{CONCLUSION}

Thermal expansion interferometry is a simple and very sensitive tool for nondestructive material analysis. In its basic form highly reflective surfaces are needed however. We developed a modified experimental set-up that can be used on any surface and found a theoretical description in good agreement with the results.

\section{ACKNOWLEDGEMENT}

This project was partly supported by the BMFT (Bundes Ministerium für Forschung und Technologie) Contract No. 1306 and the DFG (Deutsche Forschungs Gemeinschaft) Contract No. 2552. The responsibility for the contents of the paper is entirely by the authors.

The authors would like to thank Dr. W. Arnold, Prof. J. Burt and Dr. G. Busse for many helpful discussions and providing additional information.

\section{REFERENCES}

1. A.G. Bell: Am. J. Sci. 20 (1880), 305

2. R.M. White: J. Appl. Phys. 34 (1963) 3559

3. A. Rosencwaig and A. Gersho: J. Appl. Phys. 47 (1976) 64

4. W. Arnold: Acta Phys. Slov. 36 (1986), No.1

5. G. Busse: Appl. Opt. 21 (1982) 107

6. Z. Sodnik and H.J. Tiziani: Optics Comm. 58 (1986) 295

7. Y. Martin and E.A. Ash: Elect. Lett. 18 (1982) 763

8. H.S. Carslaw and J.C. Jaeger: In Conduction of heat in solids, 2nd.ed. (Clarendon Press, Oxford 1973) 